\title{
Has there been a turning point in the numbers of AIDS and HIV antibody positive cases in Ireland?
}

\author{
GE Kelly ${ }^{1}$, SM Clarke ${ }^{2}$ \\ Department of Statistics', University College Dublin, and \\ Department of Genitourinary Medicine ${ }^{2}$, St James's Hospital, Dublin, Ireland
}

\begin{abstract}
Background Major developments in the prevention and treatment of human immunodeficiency virus (HIV) infection and acquired immunodeficiency syndrome (AIDS) have taken place in recent years. Changes in the size of the HIV and AIDS epidemic need to be monitored to assess these developments and plan future services.
\end{abstract}

Aims To describe temporal trends in the incidence of HIV and AIDS in Ireland, describing separately the major risk groups, and to explore possible associations between these trends with developments in care.

Methods The annual numbers of HIV and AIDS in Ireland were analysed to determine whether there has been a turning point in incidence.

Results For AIDS, there has been an overall decrease in numbers since 1993, with both homosexual and intravenous drug users (IDU) risk groups exhibiting a decrease. For HIV antibody positive individuals, overall numbers have remained constant. However, there has been an upward trend in the heterosexual risk group and a downward trend in the IDU risk group. Thus both AIDS and HIV numbers exhibit turning points.

Conclusions Declines in HIV and AIDS incidence in the homosexual and IDU risk groups are indirectly attributable to health safety and treatment programmes. The increase in HIV antibody positive cases among heterosexuals may reflect a relapse in safe sex behaviour.

\section{Introduction}

The first cases of AIDS in Ireland were reported in 1982. ${ }^{1}$ Since then, there have been major developments in health-care both internationally ${ }^{2,3}$ and in Ireland. ${ }^{4}$ The current standard of care for the treatment of HIV infection involves initiating highly aggressive antiretroviral therapy (HAART) at an early stage of infection. Treatment usually consists of a triple combination of dual nucleoside reverse transcriptase inhibitors, with either a protease inhibitor or a non-nucleoside reverse transcriptase inhibitor. It is important to ascertain what effect these developments have had on the AIDS epidemic and to predict future trends.

The impact of prevention measures also needs to be assessed. These include the introduction of the safe sex programme by the Department of Health and Children and the introduction of needle sharing exchange centres in $1989 .{ }^{5}$

Recent reports from other countries ${ }^{6,7}$ have described changing patterns in HIV and AIDS incidence. Similar studies have not been undertaken in Ireland and therefore an overview of available data is presented here. We examined the AIDS and HIV data' for turning points, examining separately die three major risk groups: homosexuals, IDU and heterosexuals. An analysis of the total number of HIV antibody tests as reported by the Virus Reference Laboratory was also performed.

This article is a reproduction of that published in: Irish Journal of Medical Science, 169(3), pp. 183-186. Pagination may not match that of the original. 


\section{Methods \\ Statistical analysis}

The problem of determining whether there has been a change in numbers of HIV antibody positive and AIDS cases is formulated as that of locating the maximum (or minimum) of a Poisson quadratic regression. The assumption is that die number of HIV antibody positive cases in year t has a Poisson distribution with mean $\mu(t)$. The mean $\mu(t)$ is assumed to be a quadratic function of $\mathrm{t}$, i.e. $\mu(\mathrm{t})=a_{0}+a_{1} \mathrm{t}+a_{2} \mathrm{t}^{2}$. The 'goodness of fit' of the model is determined using the likelihood ratio statistic. Standard maximum likelihood estimates $\hat{a}_{0}, \hat{a}_{1}$ and $\hat{a}_{2}$ are found for the parameters $a_{0}, a_{1}$ and $a_{2}$ respectively, together with associated standard errors. A likelihood ratio test is used to determine if the parameter Oh is non-zero. If so, by differentiating the function $\mu(\mathrm{t})$, we see the turning point, denoted by to, is then given by $t_{0}=-0.5 a_{1} / a_{2}$. It is estimated by $\wedge t_{0}=-0.5 \hat{a}_{1} / \hat{a}_{2}$. If $\hat{a}_{2}$ is not significantly different from zero, then the $\hat{a}_{2}$ term is omitted from the model. Estimates of $\hat{a}_{0}$ and $\hat{a}_{1}$ are recomputed. If $\hat{a}_{1}$ is significantly different from zero, then an increasing or decreasing trend in the mean function $\mu(t)$ is indicated.

This Poisson model is also used in analysing the AIDS data. This method has been used previously in a similar study in the US. ${ }^{8}$ Fieller's theorem is used to find confidence intervals for the location of turning points. ${ }^{9}$

Linear regression was used to determine if the annual number of HIV antibody tests has changed significantly over time.

Results are reported as estimates of the turning points with associated 95\% confidence intervals. When no turning point exists, significant increasing or decreasing trends are reported.

\section{Results \\ AIDS incidence}

The numbers of AIDS cases in Ireland to December 1999, as reported to the Department of Health and Children are given in Table 1. The number of cases of AIDS together with the numbers predicted by the Poisson quadratic regression model, by year of diagnosis, is shown in Figure 1. A likelihood ratio test determined that a change point occurred in the incidence over time $(\mathrm{p}<0.0001)$. It was estimated to be July 1993 with $95 \%$ confidence interval (December 1992 to May 1994). There was an increasing trend before the change point and a decrease thereafter. For the homosexuals, the estimate was July 1993 with $95 \%$ confidence interval (June 1992 to July 1995). For the IDUs it was February 1993 with 95\% confidence interval (July 1992 to October 1993). The difference in the two groups was not statistically significant. The numbers of AIDS cases in the heterosexual risk group were too small for analysis (see Table 1).

\section{HIV incidence}

The numbers of HIV antibody positive cases to September 1999 by annual time period of diagnosis by the Virus Reference Laboratory and reported to the Department of Health and Children are shown in Table 2. When no category was identified, the risk group was declared unspecified. Due to historical reasons, the reports on HIV statistics issued by the Department of Health and Children amalgamate the figures in the heterosexual and risk unspecified groups. Separate figures are not presently available. 
Table 1: AIDS cases by risk group and year of diagnosis

$\begin{array}{llllll}\text { Category } & \begin{array}{l}\text { IV drug } \\ \text { users }\end{array} & \begin{array}{l}\text { Homo- } \\ \text { sexual }\end{array} & \begin{array}{l}\text { Hetero- } \\ \text { sexual }\end{array} & \text { Other } & \text { Total } \\ 1982 & 0 & 2 & 0 & 0 & 2 \\ 1983 & 0 & 0 & 0 & 1 & 1 \\ 1984 & 0 & 1 & 0 & 2 & 3 \\ 1985 & 1 & 1 & 0 & 3 & 5 \\ 1986 & 1 & 1 & 0 & 4 & 6 \\ 1987 & 9 & 6 & 1 & 4 & 20 \\ 1988 & 10 & 21 & 1 & 6 & 38 \\ 1989 & 21 & 17 & 2 & 11 & 51 \\ 1990 & 27 & 22 & 8 & 4 & 61 \\ 1991 & 31 & 23 & 11 & 6 & 71 \\ 1992 & 27 & 15 & 7 & 1 & 50 \\ 1993 & 42 & 13 & 11 & 2 & 68 \\ 1994 & 21 & 27 & 7 & 12 & 67 \\ 1995 & 23 & 16 & 10 & 6 & 55 \\ 1996 & 33 & 34 & 11 & 1 & 79 \\ 1997 & 9 & 12 & 7 & 4 & 32 \\ 1998 & 11 & 13 & 5 & 12 & 41 \\ 1999 & 14 & 13 & 9 & 5 & 41\end{array}$

Table 2: HIV antibody positive cases by risk group and year of diagnosis

\begin{tabular}{lllllll} 
Category & $\begin{array}{l}\text { IV drug } \\
\text { Users }\end{array}$ & $\begin{array}{l}\text { Homo- } \\
\text { sexuals }\end{array}$ & $\begin{array}{l}\text { Hetero/risk } \\
\text { Unspecified }\end{array}$ & Other & $\begin{array}{l}\text { Total positive } \\
\text { Individuals }\end{array}$ & $\begin{array}{l}\text { Total No. } \\
\text { of Tests }\end{array}$ \\
\hline $1985^{*}$ & 221 & 39 & 0 & 103 & 363 & \\
1986 & 112 & 11 & 21 & 25 & 169 & 4,743 \\
1987 & 72 & 21 & 26 & 26 & 145 & 768 \\
1988 & 58 & 17 & 20 & 20 & 115 & 8177 \\
1989 & 57 & 33 & 0 & 26 & 116 & 10,085 \\
1990 & 50 & 25 & 24 & 12 & 111 & 10,405 \\
1991 & 34 & 27 & 25 & 6 & 92 & 10,143 \\
1992 & 82 & 5 & 50 & 11 & 201 & 13,005 \\
1993 & 52 & 48 & 21 & 16 & 137 & 13,253 \\
1994 & 20 & 31 & 22 & 12 & 85 & 14,126 \\
1995 & 19 & 33 & 30 & 9 & 91 & 13,558 \\
1996 & 20 & 41 & 27 & 18 & 106 & 14,452 \\
1997 & 21 & 37 & 40 & 21 & 119 & 14,742 \\
1998 & 26 & 37 & 47 & 26 & 136 & 18,303 \\
To Sept. & & & & & & \\
1999 & 58 & 23 & 43 & 27 & 151 & 15,880 \\
$*$ *umulative & numbers & & & & &
\end{tabular}

The data were analysed, again using the Poisson quadratic regression model. The model indicated that the number of HIV positive cases has remained roughly constant over time, but not in the individual risk groups. There has been a significant upward trend in the heterosexual/risk unspecified group $(\mathrm{p}<0.01)$ and there has been a significant downward trend in the IDU risk group $(\mathrm{p}<0.002)$. In the homosexual risk group, there has been a turning point $(\mathrm{p}<0.001)$ in the number of HIV antibody positive cases. The peak is estimated to have occurred in November 1993. The data are illustrated in Figure 2, which shows the number of HIV positive individuals in each of the risk groups over time. 


\section{HIV tests}

The total number of HIV tests to September 1999 by annual time period in which the test was analysed by the Virus Reference Laboratory and reported to the Department of Health and Children is shown in Table 2. Note that a case refers to an individual, while the same individual may or may not account for more than one test.

The regression model of number of HIV tests over time showed that the number of tests has increased over time, as can be seen in Table 2. Data on the annual number of HIV antibody tests per risk group were available, only for the years 1995 to (September) 1999. The annual number of tests for each of the three risk groups being considered here has increased in this time.

In these latter years, the heterosexual/risk unspecified group has accounted for approximately $74 \%$ of tests for the three risk groups (carried out annually), and it has not changed in these five years. The cumulative proportion of positive individuals out of total tests carried out in this group is also constant, at approximately $66 \%$ in each of these five years.
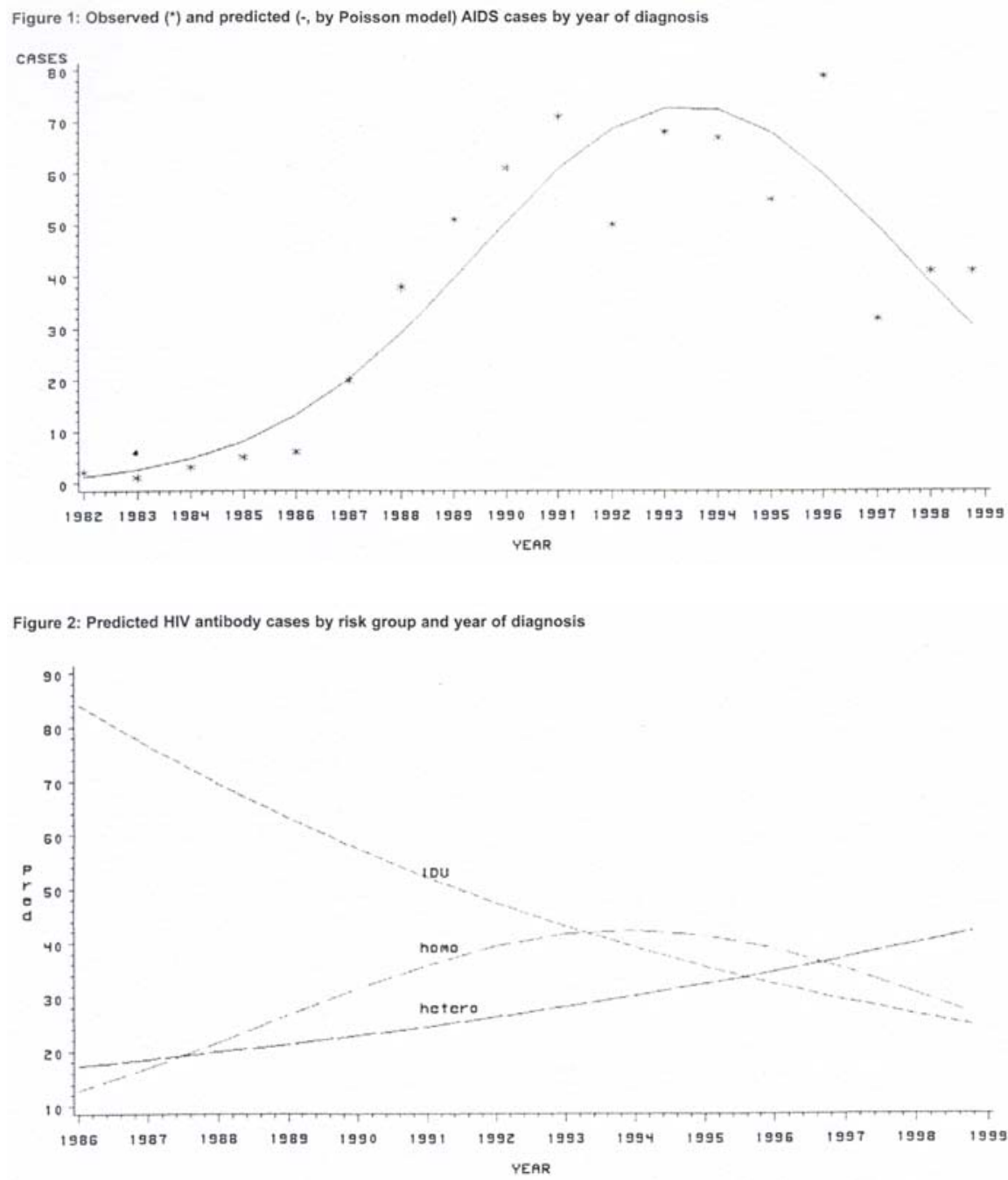


\section{Discussion}

We have found in this study that both homosexual and IDU risk groups exhibit a decrease in AIDS incidence at approximately the same time - 1993. This is consistent with reports in the UK. ${ }^{6}$ The decrease is perhaps a consequence of the use of HAART. A dramatic reduction in AIDS incidence in the US coincided with the introduction of HAART, specifically protease inhibitor therapy. ${ }^{10}$ Palella et $\mathrm{al}^{11}$ showed a dramatic reduction in the incidence of the three most common opportunistic infections, Pneumocystis carinii pneumonia (PCP), Mycobacterium avium complex (MAC) disease and cytomegalovirus retinitis (CMV) infection, from 21.9/100 person years in 1994 to 3.7/100 person years in 1997. In the UK, the increased use of HAART has been reported to be effective ${ }^{12}$ in prolonging both the incubation period of AIDS and survival in patients with AIDS.

Examination of HIV incidence rather than AIDS gives a clearer picture of trends in HIV infection. This can be argued as follows. Two measures of disease frequency, incidence and prevalence are common in epidemiology. Incidence refers to new cases of disease occurring among previously unaffected individuals. Point prevalence is that proportion of a defined population affected by the disease in question at a specified point in time. The numerator of the proportion comprises all those who have the disease at that instant, regardless of whether it was contracted recently or long ago.

In trend analysis of HIV infection, it is more appropriate to study incidence. This is because low seroincidence must be considered a requirement that an epidemic has entered a declining phase. The duration of survival of patients who arc HIV antibody positive, and hence its prevalence, may be influenced by treatment and other factors which come into play after diagnosis. Causal factors necessarily operate prior to diagnosis. In addition, AIDS incidence numbers only partially reflect HIV prevalence.

There has been no decrease in the overall numbers of HIV antibody positive cases in Ireland. This is mainly due to a recent increase among the heterosexual/risk unspecified group. Similar to the $\mathrm{UK}^{6}$ for HIV infection, new cases in heterosexuals now outnumber new cases acquired by homosexual exposure (see Table 2). This may be linked to increases in sexually transmitted diseases (STDs) among heterosexuals. Statistics from genitourinary medicine clinics ${ }^{13}$ provide the most comprehensive source of data on the epidemiology of STDs in the UK. Between 1995 and 1997, the total number of diagnoses of genital chlamydia, genital warts and Neisseria gonorrhoeae increased by $20 \%, 8 \%$ and $5 \%$ respectively. The rises have been most pronounced in 16 to 19-year-olds, both male and female. In New York City, high HIV seroprevalence has been associated with gonorrhoea in STD clinics. ${ }^{14}$ Thus, it can be argued, we may be seeing a relapse in safe sex behaviour among heterosexuals in Ireland.

We have found in this study, however, that in both the homosexual and IDU risk groups, reported HIV incidence is decreasing. The legalisation, public funding and expansion of the community-based syringe exchange programmes in New York City was associated with the large reductions in risk behaviour and HIV incidence among IDUs who used these programmes. ${ }^{7,15,16}$

It could be speculated that in Ireland also, similar prevention measures ${ }^{45}$ targeted at these risk groups, have had an impact. A study in a Dublin needle exchange reported $50 \%$ of respondents had not shared equipment in the previous 28 days. In addition, $25 \%$ of respondents used condoms always, with a further $44 \%$ using them sometimes. ${ }^{17}$ This, while not eliminating HIV transmission in the IDU group, may have slowed it down. 
The total number of HIV antibody tests carried out annually has been increasing and has doubled in the IDU and heterosexual/risk unspecified groups in recent years. This may reflect a greater health awareness in the community. It may also be that more people present for testing so that they may benefit from treatment. Counselling policies have changed in the past three years to encourage testing (whereas prior to this, they may have inhibited testing).

The annual numbers of HIV and AIDS antibody positive cases may have been underestimated for the earlier years of the epidemic. This may have been through lack of recognition by physicians unfamiliar with the condition and, in some instances, frank concealment of cases. In the UK, an estimated $13 \%$ of AIDS cases go unreported. ${ }^{18}$ Thus the declines in incidences reported in this study may be indicative of even larger reductions.

\section{Conclusion}

HIV infection in Ireland is a cause for concern. A total of 2,137 positive individuals were reported to September 1999 - about 125 new cases reported per year in recent years. Most new diagnoses are recent seroconversions (Dr Clarke - clinical practice observation). This indicates a turning away from safe sex practices in the heterosexual/risk unspecified group and unsafe needle practices in the IDU group. No information is currently available on whether the heterosexual/risk unspecified group acquire their infection locally or abroad, and/or from risk group partners. Further studies on these issues would be helpful in identifying appropriate prevention measures.

Medical advances have slowed the progression of HIV disease but new infection remains a significant problem. None of the currently available treatments are able to completely eradicate the virus. Long-term treatment impairs the lives of sufferers and places a significant financial demand on medical resources. ${ }^{4}$

\section{References}

1. Department of Health and Children, Ireland. HIV/AIDS Quarterly Reports.

2. Carpenter CJ, Fischi NA, Hammor SM et al. Antiretroviral therapy for HIV infection in 1998; updated recommendations of the International AIDS Society - USA panel. JAMA 1998; 280: 78-86.

3. Staszewski S, Miller V, Sabin C, Carleback A, Berger AM et al. Virological responses to protease inhibitor therapy in a HIV clinic cohort. AIDS 1999; 13: 367-73.

4. Bradley F, Bury G, O'Kelly F et al. Analysis of care of HIV positive patients: hospital and general practice components. Ir Med J 1995; 88: 98-100.

5. Bury G, O'Kelly F, Pomeroy L. The use of primary care services by drug users attending a HIV prevention unit. Ir Med J 1993; 86: 53-5.

6. European Centre for the Epidemiological Monitoring of AIDS. HIV/AIDS Surveillance in Europe, Quarterly Reports. St Maurice, France.

7. Des Jarlais DC, Marmor M, Friedmann $P$ et al. HIV incidence among injection drug users in New York City, 1992-1997: evidence for a declining epidemic. Am J Pub Hith 2000; 90: 352-9.

8. Zhou S, Kingley L, Taylor J, Chmiel J, He D, Hoover D and The Multicentre AIDS Cohort Study. A method to test for a recent increase in HIV-1 seroconversion incidence: results from the Multicentre AIDS Cohort Study (MACS). Stat in Med 1993; 12: 153-64.

9. Finney DJ. Probit Analysis, Cambridge: Cambridge University Press, 3rd edition, $1971 ; 78$.

10. Plexner C. HIV protease inhibitors. N Engl J Med 1998; 338: 1281-91.

11. Palella FJ, Delaney KM, Moorman AC, Loveless MO, Fuhrer J, Holmberg SD. Declining morbidity and mortality among patients with advanced HIV infection. $N$ Engl $J$ Med 1998; 338: 853-60. 
12. Report of an Expert Group (Chairman: Professor Day NE). The incidence and prevalence of AIDS and prevalence of other severe HIV disease in England and Wales for 1995 to 1999; projections using data to the end of 1994. Commun Dis Report, CDR Review 1996; 1: R1-R24.

13. Hughes G, Simms I, Rogers PA, Swan AV, Catchpole M. GUM statistics. Commun Dis Report, CDR Suppl 1998; 7: Sl-11.9.

14. Torian L, Makki H, Menzies I et al. High HIV seroprevalence associated with gonorrhea: New York City Department of Health, sexually transmitted disease clinics, 19901997. Aids 2000; 14: 189-95

15. Des Jarlais DC, Marmor M, Paone D et al. HIV incidence among injecting drug users in New York City syringe exchange programmes. Lancet 1996; 348: 987-91.

16. Des Jarlais DC, Perlis T, Friedman SR et al. Risk reduction in a declining HIV epidemic: injecting drug users in New York City, 1990-1997. In press.

17. Johnson Z, O'Connor M, Pomeroy L et al. Prevalence of HIV and associated risk behaviour in attendees at a Dublin needle exchange. Addiction 1994; 89: 603-7.

18. Molesworth A. Results from the 1995 survey of prevalent clinically diagnosed HIV infection in England, Wales and Northern Ireland. Commun Dis Report, CDR Review 1997; 6: R77-R82 